\title{
Estimation of electric charge in sprites from optical and radio observations
}

\author{
Jingbo $\mathrm{Li}^{1}$ and Steven Cummer ${ }^{1}$ \\ Received 22 February 2010; revised 6 September 2010; accepted 14 October 2010; published 8 January 2011.
}

[1] Measurements of very low frequency radio emissions indicate that substantial electric current flows inside some sprites. This charge motion, with presently unknown location and distribution, is related to the detailed internal microphysics of sprite development that is in turn connected to the impact sprites have on the mesosphere. Assuming that sprite streamers propagate along the direction of local electric fields, we combine lightningdriven background electric fields computed from electromagnetic measurements and simulations with streamer timing and propagation direction measured from high-speed images to estimate the total ambient electric field at the locations of upward streamers. From this total field, we are able to establish a lower bound on the electric charge in six observed sprites driven by positive lightning. We find that individual bright sprite cores contain significant negative space charge between $-0.01 \mathrm{C}$ and $-0.03 \mathrm{C}$. Accounting for the uncertainty caused by using different vertical charge distribution profiles, these values could be as much as $50 \%$ higher. This charge, when spread over the observed sizes of several hundred meters in diameter and approximately $10 \mathrm{~km}$ in length, results in a local electric field close to the breakdown field and that the relatively long duration (several milliseconds) optical emissions from this region are driven predominantly by instantaneous local electric fields, in agreement with published simulation results. Given the significant negative charge, we interpret the sprite core region as the partial and perhaps dominant sink of the negative charge created by the downward positive polarity streamers. This further suggests that when downward streamers supply more charge than can be absorbed by the sprite core, slightly delayed upward negative streamers initiate from the sprite core to disperse this charge. This is consistent with observations that show that the subsequent upward streamers are not always present, especially in smaller sprites. Assuming that the sprite core balances all of the positive charge in the downward streamers, we find that the average streamer contains a typical charge of at least $2-4 \times 10^{-3} \mathrm{dC}$. Observed sprite current in one bright sprite suggests a positive charge in each distinct downward streamer of only 2.5 times larger than this lower bound, indicating that our estimate of the positive charge in downward sprite streamers and the negative charge in the sprite core is physically reasonable.

Citation: Li, J., and S. Cummer (2011), Estimation of electric charge in sprites from optical and radio observations, J. Geophys. Res., 116, A01301, doi:10.1029/2010JA015391.

\section{Introduction}

[2] Sprites are structured optical emissions caused by lightning discharges and are observed at altitudes of $\sim 40$ $90 \mathrm{~km}$ above thunderstorms [Sentman, 1995]. Previous studies involving measurements of sprite optical and radio emissions have revealed many important features of the phenomena. Images recorded by high-speed cameras [Stanley et al., 1999; Stenbaek-Nielsen et al., 2000; Cummer et al., 2006; McHarg et al., 2007; Stenbaek-Nielsen and McHarg,

\footnotetext{
${ }^{1}$ Department of Electrical and Computer Engineering, Duke University, Durham, North Carolina, USA.

Copyright 2011 by the American Geophysical Union. 0148-0227/11/2010JA015391
}

2008] and telescopes [Gerken et al., 2000] have shown the fine streamer-based structure of sprites [Pasko, 2007]. Observed distinct features in sprites typically include downward streamers, an upper bright core in the region where the downward streamers initiated, and in some sprites upward streamers that develop approximately $1 \mathrm{~ms}$ after the downward streamers [Stanley et al., 1999; Cummer et al., 2006].

[3] Laboratory experiments [Ebert et al., 2006; Luque et al., 2008; Briels et al., 2008] and numerical models [Liu and Pasko, 2004, 2005; Sentman et al., 2008; Luque and Ebert, 2009, 2010] have shown that upward and downward streamers are formed by the motion of electrons in an ambient electric field. The polarity of these streamers is defined by a sign of the charge in its head and related to the polarity of the sprite-producing lightning discharge [Liu and 
Pasko, 2004]. Chemical processes during sprite development have been studied to explain the brightness of sprite streamers [Morrill et al., 1998; Stenbaek-Nielsen et al., 2007; Sentman et al., 2008]. The longer-duration optical emissions from the bright sprite core are less understood and could reflect either slow local chemical processes [McHarg et al., 2007] or long duration local electric fields.

[4] Studies from remote measured low-frequency radio emissions indicate that substantial electric current can flow inside the sprite body during an optical emission [Cummer et al., 1998]. This electric current, known as "sprite current," is related to internal microphysics of sprite development and is also connected with the effects sprites create in the mesosphere. However, it has been difficult to determine where this current flows inside a sprite. Numerical models have been used to predict the sprite properties including current flows and charge motion in sprites. Raizer et al. [1998] modeled the sprite streamer propagation in an inhomogeneous atmosphere by treating the lightning source as an electrical dipole. Including the internal chemical and photoionization processes, Liu and Pasko [2004, 2005] have developed a model to simulate the propagation of sprite streamers in uniform electric fields and air densities. Luque and Ebert [2009, 2010] reported simulation results of streamer propagation in a varying air density. Besides properties of propagating positive streamers, recent simulation results from Liu [2010], Luque and Ebert [2010] also revealed the mechanism of the glowing trail behind, which is produced by the accumulation of negative charges. All these models have been proven to be useful in predicting the sprite dynamics at the beginning stage of the sprite development and have been confirmed with observations from high-speed videos [McHarg et al., 2007; Cummer et al., 2006; Li and Cummer, 2009; Liu et al., 2009b; Luque and Ebert, 2009]. However, model predictions depend very sensitively on the input parameters and initial conditions. Furthermore, it can be difficult to simulate the sprite properties at the stage of full development due to the limitations imposed by computation resources. Thus measurements of or bounds on the total charge in sprites are needed to help constrain detailed streamer models and thus improve predictions of the physical and chemical impact of sprites on the mesosphere.

[5] In this work, we estimate the total electric charge in sprites using a measurement-based approach. First, we estimate the lightning-driven background electric fields in the mesosphere by combining measurements of lightning radiated magnetic fields and detailed electromagnetic simulations [Hu et al., 2007; Li et al., 2008]. We then measure from high-speed video observations the propagation direction of upward streamers that initiate from the bright core region of sprites. Assuming the streamers propagate along the direction of local electric fields, and that the local electric fields are modified by space charge present in the sprite core, the above analysis approach enables us to infer the total electric charge in the bright core region that is consistent with the observed streamer propagation directions.

[6] We apply this analysis approach to six sprites that were driven by positive lightning. We find that individual bright sprite cores contain significant negative space charge that ranges over a factor of 4 between $-0.009 \mathrm{C}$ and $-0.034 \mathrm{C}$. Considering the uncertainty caused by using different charge distribution profiles, this charge may be as much as $50 \%$ larger and thus should be considered a lower bound. Detailed electrostatic simulations show that this amount of charge, when spread over the observed sizes of several hundred meters in diameter and approximately $10 \mathrm{~km}$ in length, results in a local electric field at the outer edges of the sprite core close to the air breakdown field. This is consistent with the recent simulation results reported by Luque and Ebert [2010]. Moreover, this indicates that the relatively long duration (several milliseconds) optical emissions typically observed from sprite cores [Cummer et al., 2006] are driven predominantly by instantaneous local electric fields and not by long-duration chemistry initiated by previous energy input.

[7] Given the significant negative charge, we interpret the sprite core region as the partial and perhaps dominant sink of the negative charge created by the downward positive polarity streamers. This notion is supported by numerical simulations which show that only a weak or nonexistent electrical connection between the ionosphere and the downward streamer results in significant negative charge at the top of the streamer channel. This further suggests that subsequent negative polarity upward streamers that appear in some sprites a few milliseconds after initiation are created when the downward positive sprite streamers require more charge than can be supplied by this sprite core. This is consistent with observations that show the subsequent upward streamers are not always present, especially in smaller sprites.

[8] Assuming that the sprite core balances all of the positive charge in the downward streamers, we find that the average downward streamer contains a charge of at least $2-4 \times 10^{-3} \mathrm{C}$. Observed sprite current in one bright sprite suggests a positive charge in each distinct downward streamer that is only 2.5 times larger than this lower bound. This indicates that our estimate of the positive charge in downward sprite streamers and the negative charge in the sprite core is physically reasonable, and also that the charge motion from the sprite core to the downward streamers is a likely source of sprite current.

\section{Instrumentation}

[9] In 2005 we simultaneously measured high-altitude sprite optical emissions and broadband electromagnetic radiation from sprite parent lightning at the Yucca Ridge Field Station $\left(40.702^{\circ} \mathrm{N},-105.031^{\circ} \mathrm{E}\right)$ near Fort Collins, Colorado, USA. The magnetic sensors continuously sampled signals with a flat response from $\sim 2 \mathrm{~Hz}$ to $30 \mathrm{kHz}$. Additionally, the National Lightning Detection Network (NLDN) data provided all of the return stroke locations. The lightning location and two orthogonal horizontal measurements of the magnetic field from each pair of the coils enabled us to derive the azimuthal magnetic field (the magnetic field in azimuthal $(\hat{\phi})$ direction defined by a cylindrical coordinate system with the origin at the lightning location). The camera used was a Vision Research Phantom 7.1 monochrome highspeed imager coupled to an ITT Gen III image intensifier, and during the campaign we recorded high-speed TLE images up to 10,000 frames per second. An external GPS-synchronized IRIG time code has been used to provide the absolute timing for both systems with an accuracy better than 10 microseconds. Thus the resulting data set comprises synchronized, 


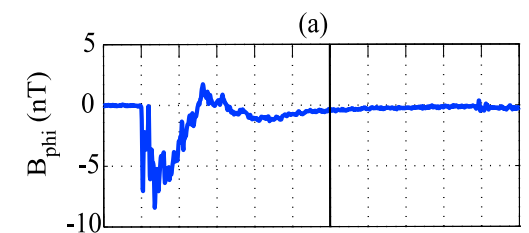

(b)
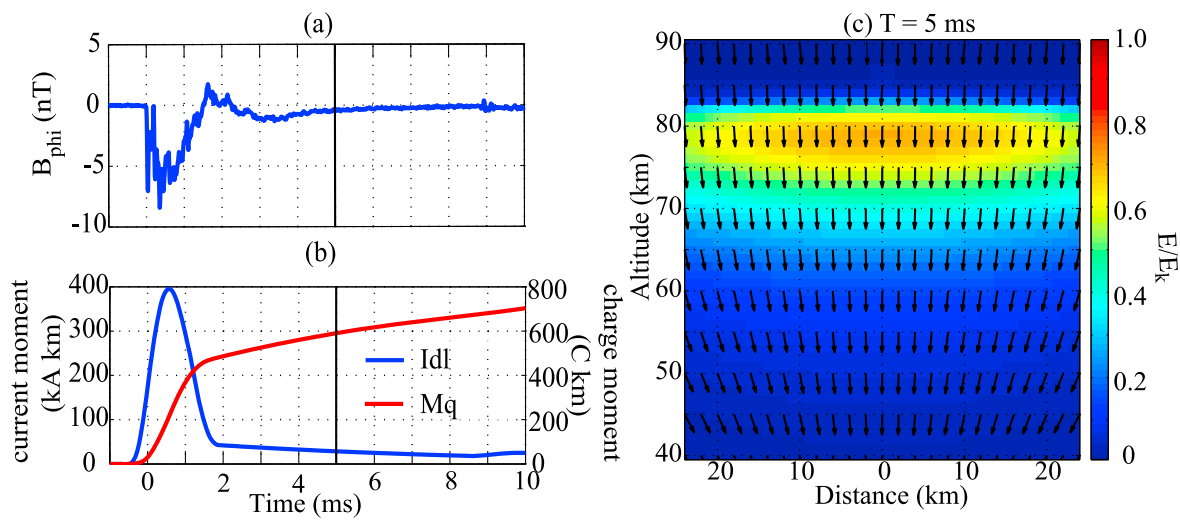

Figure 1. An example of background electric field computation. (a) Magnetic field measured from a sprite-producing +CG. (b) Extracted current moment and charge moment change. (c) FDTD simulation results of lightning-driven electric field at $5 \mathrm{~ms}$ after the return stroke. The color intensity represents the normalized electric field $\left(E / E_{k}\right)$. The arrows represent the direction of the electric fields.

high time resolution images and broadband magnetic field measurements. More details about these instruments were presented by Cummer et al. [2006] and Li et al. [2008].

\section{Method of Analysis}

[10] The main element of our analysis is inferring the total charge in the sprite core region from observations of the direction of travel of upward streamers that emanate from the core. To do this we must separately estimate the contributions to the total mesospheric electric field at the location of these upward streamers from the low-altitude lightningdriven fields and from local charge inside the sprite core.

\subsection{Lightning-Driven Mesospheric Electric Fields}

[11] We first estimate the lightning-driven electric fields by applying a method introduced by $\mathrm{Hu}$ et al. [2007] and Li et al. [2008], which combines a deconvolution technique [Cummer and Inan, 1997; Cummer, 2003] and finite difference time domain (FDTD) simulations [Hu et al., 2006]. The deconvolution technique is used to infer the lightning current moment waveform from the remotely measured magnetic fields. This source current is then used as an input to the FDTD model. The FDTD model computes electric fields as a function of both time and location above the thundercloud. Parameterized nonlinear heating, attachment, and ionization processes [Pasko et al., 1997] are included in this model and are essential for accurately estimating lightning-driven mesospheric electric fields. Strong lightningdriven fields create significant electron attachment that reduces the local electron density and thus increases the local electric field by reducing relaxation currents. Not including these nonlinearities can result in predicted field differences as big as a factor of two near the $\sim 70-80 \mathrm{~km}$ altitude of interest. All of the sprites analyzed here are produced by positive cloud-to-ground lightning discharges (+CGs), and we use one example to illustrate our approach. Figure 1 shows one example of lightning generated electric fields using the technique described by Hu et al. [2007] and Li et al. [2008]. Figure 1 (left) shows the measured magnetic fields and extracted current moment waveform. Figure 1 (right) shows a snapshot of lightning produced electric fields at a typical sprite delay time ( $5 \mathrm{~ms}$ in this case) after the return stroke. The color intensity represents the normalized electric field, which is the ratio between the true electric field $E$ and air breakdown field $E_{k}$. The black arrows represent the direction of this lightning induced electric field. The direction of the lightning-driven field after the return stroke is not sensitive to the delay time due to its quasi-electrostatic nature. In the examples that follow, the time at which the background electric field is computed is the time at which the upward streamer develops according to high-speed images.

[12] In this way we can realistically estimate the lightningdriven mesospheric electric field for each observed sprite. As shown in this case, it is generally true that at locations above the lightning discharge above approximately $60 \mathrm{~km}$, the lightning-driven electric field is very nearly vertical and in the analysis that follows, we neglect the small horizontal component. The essentially vertical lightning-generated field means that any deviations from vertical in upward streamer development must be created by local charge in the mesosphere.

\subsection{Local Mesospheric Electric Fields From Sprites}

[13] If we assume that streamers propagate along the direction (or opposite direction for negatively charged streamers) of the total electric field, the total electric field and electric fields caused by charges in sprites can be computed by knowing the lightning-driven fields and streamer propagation directions, which can be measured from high-speed videos. Figure $2 \mathrm{a}$ shows a single still image acquired at 7200 frames per second of a typical and relatively small sprite caused by a $+\mathrm{CG}$ with labeled downward streamers, bright core, and upward streamers. The upper part of this sprite is zoomed in Figure $2 b$ to show the detailed structure.

[14] The upward streamers are negative polarity since they move opposite to the general electric field direction [Stanley et al., 1999; Cummer et al., 2006]. However, as seen in the image, upward streamers propagate with a significant horizontal component [Cummer et al., 2006; McHarg et al., 2007] that we assume is produced by a horizontal component of the local electric field. Since the lightning-driven field is vertical, this horizontal field has to be generated by local electric charge associated in some way with the sprite. 
(a)

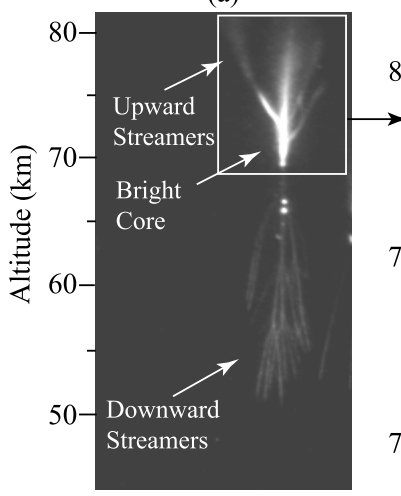

(b)

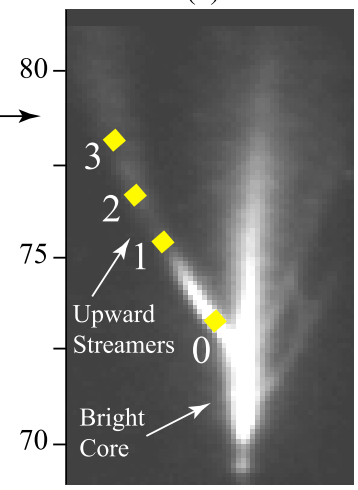

(c)

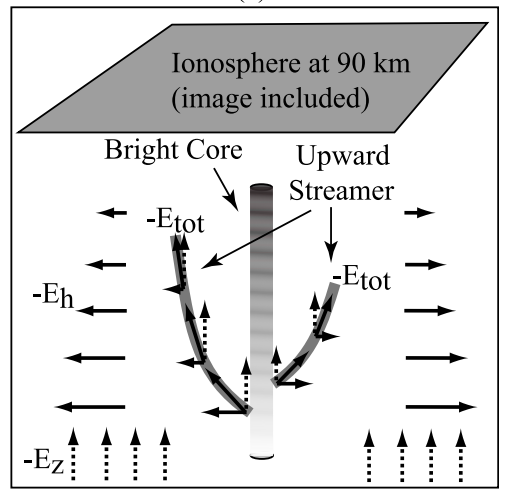

Figure 2. (a) Image of a typical sprite including downward streamers, a bright core, and upward streamers. (b) Zoomed sprite upper part to show the bright core region and upward streamers. (c) A schematic showing the model applied and the electric field components at upward streamer locations. $E_{h}, E_{z}$, and $E_{t o t}$ represent the horizontal, vertical, and total electric field. The arrow lines represent the opposite direction of electric fields, which is the moving direction for negative charges.

The closest and brightest sprite feature is the bright core, and we thus assume that this region is the source of the needed charge. It will be shown below that this assumption is consistent with the size and brightness of this particular sprite feature. This general model is summarized in Figure 2 (right), in which the bright core and upward streamers are negatively charged and represented by a $3-\mathrm{D}$ cylinder and the two gray curves. $E_{z}, E_{h}$ and $E_{\text {tot }}$ represent the vertical electric field, the horizontal electric field, and the total electric field. Note that in Figure 2 (right) the arrows point opposite to the electric field to indicate the direction of negative polarity streamer motion. With this model, the horizontal electric field can be computed by knowing the vertical lightning-driven field and the streamer propagation direction.

\subsection{Estimating Charge in the Sprite Core}

[15] By knowing the horizontal electric field along the path of the upward streamers, we can bound the total amount of charge in the bright core from which the negative streamers initiate. In our model, this region is approximated by a thin, negatively charged circular cylinder with a varying charge in both the radial direction (or horizontal direction) and the vertical direction. The charge distribution in the radial direction and the radius of this charge distribution are not that important because for distance greater than the radius of the thin cylinder, the thin cylinder is nearly equivalent to a line source. Previous studies have revealed that the glowing trail after positive streamers is close to a conductor [Luque and Ebert, 2010]. Thus, more negative charge should appear at a surface layer of the bright conducting core than in the center. To approximate that, we choose an exponential distribution in the horizontal direction with zero charge at the center and more than $50 \%$ in a $50 \mathrm{~m}$ thick surface layer. Determining the vertical charge distribution is a challenge since there is no existing charge distribution reported for the stage when the entire sprite is fully developed. Thus we combine sprite optical emissions and previous simulation results at the beginning stage of sprite development [Luque and Ebert, 2010] to determine this vertical charge distribution. Features of the optical emissions enable us to estimate a reasonable vertical charge distribution along the sprite core. Figure $2 \mathrm{~b}$ shows that significant optical emissions are present along the entire vertical extent of the sprite core (except near its very top portion where the optical emission transitions to highly diffuse [Pasko and StenbaekNielsen, 2002]). This indicates that normalized electric field $\left(E / E_{k}\right)$ is not significantly less than unity at all altitudes. We thus choose an exponential function to approximate the vertical charge distribution in the bright core so that the charge density and $E_{k}$ decay equally with altitude to ensure a nearly constant normalized field at different altitudes. Since the inverse scale height of air density drop is $0.15 \mathrm{~km}^{-1}$ [Pasko et al., 1997], the charge density is thus given by

$$
\rho_{\text {core }}(h)=\rho_{0} \exp (-0.15 h) \text {. }
$$

Luque and Ebert [2010] has reported numerical simulation results at the beginning stage of the sprite development, at which the glowing trail expands during the propagation of downward streamers. These simulation results show that at $2 \mathrm{~ms}$ after the initiation of a positive streamer, the negative charge amount in the glowing trail along the altitude is between uniform and exponential decay with an inverse scale height of $0.25 \mathrm{~km}^{-1}$. In this work, we focused on the stage of full development, at which the bright core is static. Although not at the same stage of sprite development, the inverse scale height estimated from the optical emission is between the values from the simulation results. In this work, we choose the exponential delay with an inverse scale height of $0.15 \mathrm{~km}^{-1}$ as the vertical charge distribution to estimate the amount of charge in the bright core in the following examples. However, the uncertainty in the amount of charge caused by a uniform or an exponential charge distribution with the inverse scale height of $0.25 \mathrm{~km}^{-1}$ will be discussed in detail in section 4.1.1. With the assumed distribution, the total charge amount can be expressed by

$$
Q_{\text {core }}=\int_{h_{\text {bot }}}^{h_{\text {top }}} \rho_{\text {core }}(h) d h,
$$


Table 1. Estimated Horizontal Electric Field and Total Amount of Charges in the Bright Core ${ }^{\mathrm{a}}$

\begin{tabular}{cccccc}
\hline $\begin{array}{c}\text { Streamer } \\
\text { Location }\end{array}$ & $\begin{array}{c}\text { Altitude } \\
(\mathrm{km})\end{array}$ & $\begin{array}{c}\text { Distance } \\
(\mathrm{km})\end{array}$ & $\begin{array}{c}E_{z} \text { (Lightning) } \\
(\mathrm{V} / \mathrm{m})\end{array}$ & $\begin{array}{c}E_{r}(\mathrm{Core}) \\
(\mathrm{V} / \mathrm{m})\end{array}$ & $Q_{\text {core }}(\mathrm{C})$ \\
\hline 0 & 73.1 & 0.6 & 48.2 & 32.1 & -0.010 \\
1 & 75.0 & 1.9 & 38.4 & 23.6 & -0.026 \\
2 & 76.7 & 2.9 & 29.3 & 8.0 & -0.020 \\
3 & 78.1 & 3.3 & 25.3 & 5.4 & -0.023 \\
\hline
\end{tabular}

${ }^{\mathrm{a}} E_{z}$, the vertical electric field caused by lightning discharge; $E_{r}$, the estimated horizontal electric fields produced by charges in the bright core; $Q$, the total amount of charge inside the bright core.

where $\rho_{\text {core }}$ is the vertical charge density in $\mathrm{C} / \mathrm{m}, h$ is the altitude in $\mathrm{km}$. The altitudes of the top $\left(h_{\text {top }}\right)$ and the bottom $\left(h_{\text {bot }}\right)$ of the sprite core are measured from high-speed images. With the charge distribution known, we can compute the electric fields in radial and vertical directions produced by static charges inside the bright core. In our model, a conducting ionosphere is set at $90 \mathrm{~km}$ altitude. Thus the image of the bright core has been included when computing the fields. The electric fields caused by the bright core are linearly proportional to the total core charge $Q_{\text {core }}$ that we wish to estimate.

[16] By varying the one free parameter in the above model, $\rho_{0}$ or equivalently $Q_{\text {core, we match the radial electric }}$ field estimated from the streamer propagation direction and thereby obtain the total amount of charge in the sprite core. Table 1 shows the total sprite core charge $Q_{\text {core }}$ required to match the inferred horizontal electric fields at the four different streamer tip locations shown in Figure 2. Despite the inevitable noise in the measurements, the four independently computed values of $Q_{\text {core }}$ for this one upward streamer agree extremely well. The total sprite core charge is negative, as expected and discussed above, and is approximately $-0.02 \mathrm{C}$. The one measurement that disagrees slightly (a factor of 2) with the others is based on the location where the upward streamer initiates, which is very close to the bright core and thus the assumptions made in our model are expected to be the least valid in this location. However, for horizontal distances at least a few $\mathrm{km}$ away from the bright core, the three measurements of the total charge agree within $25 \%$, which validates the basic approach used. It should be mentioned that the bright core region also produces a vertical electric field, which contributes to the total electric field at the streamer locations. However, in our examples, the vertical field produced by the core is less than $20 \%$ of the lightning-driven fields at streamer locations. Although less important when comparing to the lightning-driven field, this field is still included by iterating the computations until a match is found between the total electric field direction and the streamer propagation direction.

[17] This approach is easiest to apply to relatively simple sprites in which individual cores and upward streamers are well-defined. In big and complex sprites it is difficult to apply. However, those sprites often contain measurable sprite current [Cummer, 2003] enabling an estimate of the charge involved. In section 4.2, these two approaches are compared.

\subsection{Source of Electric Charge in Sprites}

[18] Having established a method to estimate the negative space charge present in the core region of sprites, we now discuss the possible origin of this charge and the connection between charges in the upper bright core and in the lower downward streamers. Some previous models [e.g., Raizer et al., 1998; Liu and Pasko, 2004, 2005] treat the conducting ionosphere as an electrode so that the electric charge in the sprite body is provided by the ionosphere through the ionized channels and the channels themselves, i.e., the sprite core, is charge neutral. The example above shows that this region is not charge neutral. However, our model illustrated in Figure 2 simply enables computation of this charge and does not assume anything about the degree of electrical connection to the ionosphere and thus does not directly relate the negative charge in the core to the positive charge in the downward streamers. Here we examine this issue through numerical simulations.

[19] Figure 3a shows a scenario after a +CG lightning return stroke but before the initiation of sprites. The simulation domain is a 2-D cylinder with axial symmetry. Between $40 \mathrm{~km}$ and $90 \mathrm{~km}$ altitudes, the electrical potential is set as $1100 \mathrm{kV}$, which is computed from a measured lightning signal and FDTD simulations. To simplify the problem, we use free space as the environment instead of the inhomogeneous mesosphere, which will not qualitatively change the results. The color intensity represents the electric potential and the arrow lines represent directions of
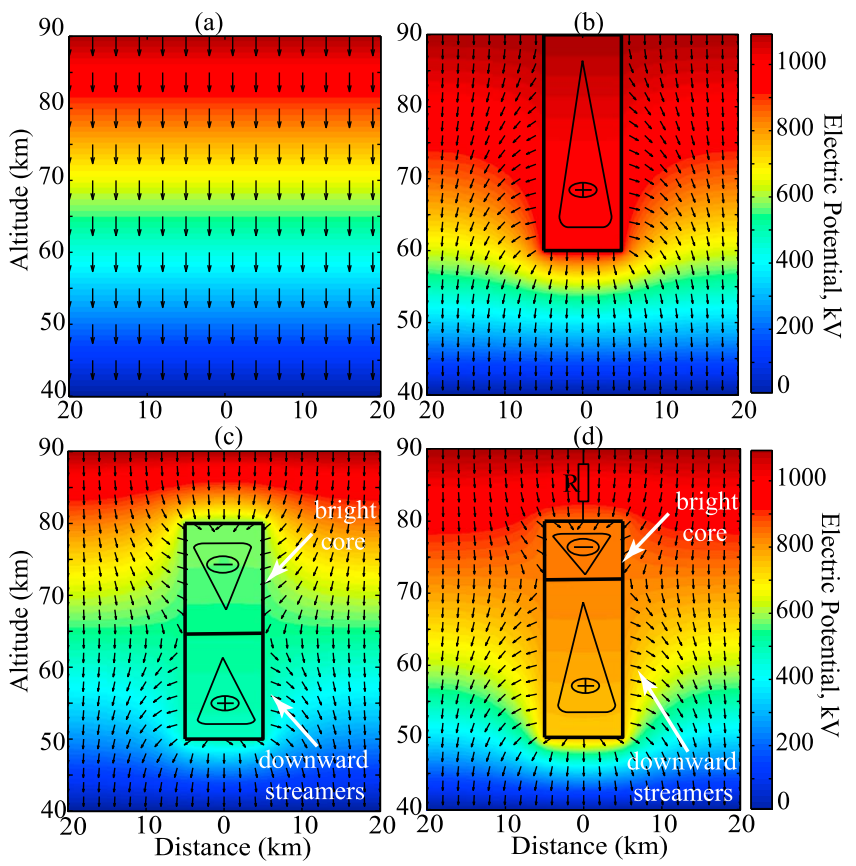

Figure 3. The electrical potential and electric field direction: (a) without a 3-D conducting cylinder; (b) the conducting cylinder is perfectly connected to the ionosphere boundary; (c) the conducting cylinder is not connected to the ionosphere boundary; (d) the conducting cylinder is partially connected to the ionosphere boundary through a resistor. 
the local electric field. Similar to the results shown in Figure $2 \mathrm{a}$, the electric fields point downward due to the fact that lower altitude has lower potential (negatively charged thundercloud).

[20] We next approximate the sprite body by a $30 \mathrm{~km}$ long conducting cylinder and insert it into the computation domain. Figure $3 b$ shows the scenario when this cylinder is perfectly connected to the ionosphere boundary at $90 \mathrm{~km}$ altitude. Under this condition, the conducting cylinder will have the same electrical potential as the ionosphere due to its high conductivity. Thus, current flows from the ionosphere to the cylinder and leaves it positively charged in the entire region. Since the cylinder is approximated by a conductor, the electric charges are distributed on the surface of the cylinder. The shape of the triangle schematically represents the amount of charge along the surface of the cylinder. The polarity is also represented by the sign inside. The horizontal electric field created by these surface charges points away from the center. However, as shown earlier, the upward streamer propagation requires the local electric field direction pointing to the sprite. Therefore this disagreement implies that the sprite cannot be perfectly connected to the ionosphere.

[21] Next we show the scenario where the conducing cylinder is not connected to the ionosphere. In Figure 3c, there is no charge transfer between the cylinder and the ionosphere, so the sprite has a zero net charge. However, free charges inside the cylinder will redistribute to maintain a zero electric field inside. This results in the top half the cylinder being negatively charged to form the bright core region while the bottom half the cylinder being positively charged to form the region of downward streamers. The electric field direction changes from pointing inward to pointing outward along the cylinder from the top to the bottom. In the vicinity of the bright core, the electric field direction can satisfy the upward streamer motion direction.

[22] We also examine a scenario between the two circumstances of perfectly connected and unconnected above. Figure $3 \mathrm{~d}$ shows the simulation result when the sprite is partially connected to the ionosphere through a resistor. In this case, current still flows from the ionosphere to the conducting cylinder but with a smaller amplitude due to the resistance. This results in positive net charges in the entire cylinder. However, before reaching the steady state, the top part of the cylinder still contains negative charges to form the bright core and the bottom part of the cylinder contains positive charges to form the region of downward streamers. Thus the electric field direction close to the bright core still can match the upward streamer motion direction.

[23] These simulation results indicate that there must be negative charges at the top part of the sprites to produce electric field in the opposite direction of streamer motion. This negatively charged region is the bright core in sprites. To accomplish that, the sprite has to be unconnected (Figure $3 \mathrm{c}$ ) or partially connected (Figure $3 \mathrm{~d}$ ) to the ionosphere. For a given sprite, our method introduced above can be used to infer the amount of negative charge within the bright core no matter if the real situation is $3 \mathrm{c}$ or $3 \mathrm{~d}$. If the scenario in Figure $3 c$ is true, the amount of this negative charge is the total charge inside the sprite body since the net charge is zero. While if the scenario in Figure $3 \mathrm{~d}$ is the real situation, the amount of this negative charge is the lower bound of the total charge since there are current flows from the conducting ionosphere, which can neutralize part of the negative charge in the core region.

\section{Results}

\subsection{Simple Sprites}

[24] We now apply our approach to estimate charge in relatively simple sprite elements produced by four different lightning discharges. Figure 4 shows the full analysis of the sprite observed on 13 August 2005, 0343 UT. Although this event has been partially described as the example in section 3.3, we provide the full details of this event in Figure 4 for completeness. Figure 4 (top) shows the portions of the high-speed sprite images recorded at 7200 frames per second that show just the upper part of the sprite. The time delay in milliseconds from the parent lightning return stroke is labeled at the bottom of each image. This sprite contains only one upward streamer on the left side of the bright core, and there is likely minimal interaction with streamers on the right side. Although the structure is relatively simple, it still contains all the features of a typical sprite like downward streamers and the bright upper core that develop simultaneously, and upward streamers that develop about $1 \mathrm{~ms}$ later.

[25] During the development of the upward streamer, the shape and size of the sprite core do not change appreciably, and we thus assume the charge distribution in the core is static. The bright core has a height of $8 \mathrm{~km}$ and an apparent radius of $300 \mathrm{~m}$ in these high-speed images, although we emphasize that the core radius does not affect our calculation of the total core charge. The upward streamer initiates about 37 degrees from vertical and then bends toward the vertical direction during propagation. Figures $4 \mathrm{~b}$ and $4 \mathrm{c}$ show the measured azimuthal magnetic field, extracted current moment waveform and charge moment change history. The impulse charge moment change (iCMC) within the first $2 \mathrm{~ms}$ of lightning return stroke is $\sim 310 \mathrm{C} \mathrm{km}$, which is consistent with observations of the charge moment change in modest short delayed sprites [Cummer and Lyons, 2005; $\mathrm{Hu}$ et al., 2007]. Based on the extracted lightning source current, we computed lightning-driven electric fields, horizontal electric fields produced by the sprite core, and total amount of charge, which have been shown in Table 1. After iterations with initial values, a best match between electric field direction and streamer motion direction is achieved at $Q_{\text {core }}=-0.02 \mathrm{C}$. Figure $4 \mathrm{~d}$ shows the total electric fields in the vicinity of the bright core. Since the upward streamers are negative polarity, the direction opposite to the local electric field is shown to indicate streamer propagation direction. At each location of streamer tips, the streamer propagates along this opposite direction of the total electric fields.

[26] Figure 5 shows the simulation results for three more sprites that occurred on different days. Figure 5 (top) shows individual high-speed images to show the relatively simple upward streamer development. Figure 5 (bottom) shows the amplitude and direction of the inferred total electric field. These three sprites are morphologically similar to the example shown in Figure 4. From each we extract sprite core charges of $-0.009,-0.011$, and $-0.016 \mathrm{C}$. The overall range from these four sprites varies by only a factor of 2 . 
(a)

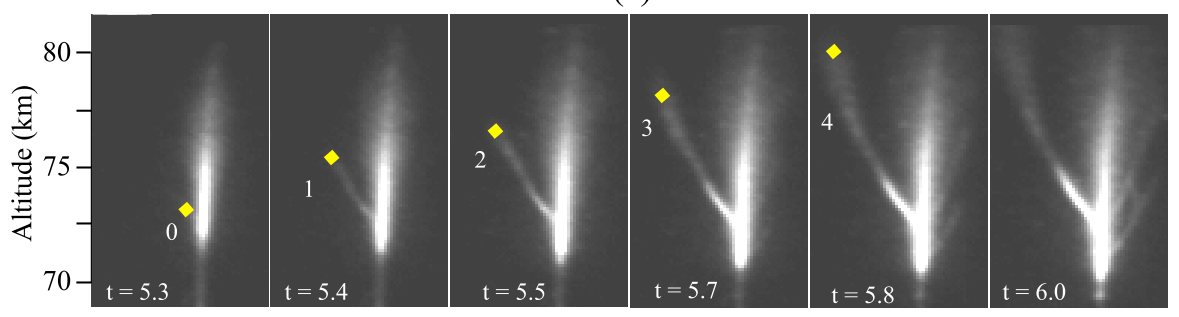

(b)

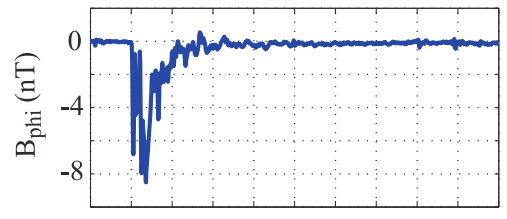

(c)

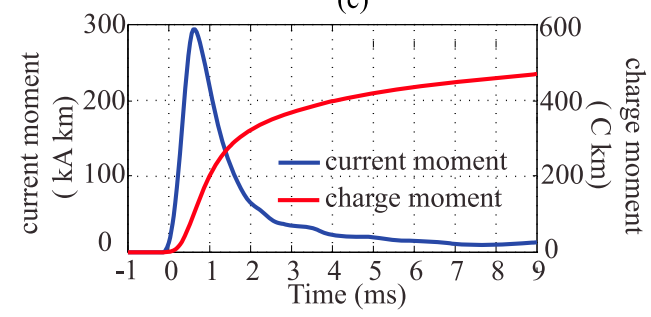

(d)

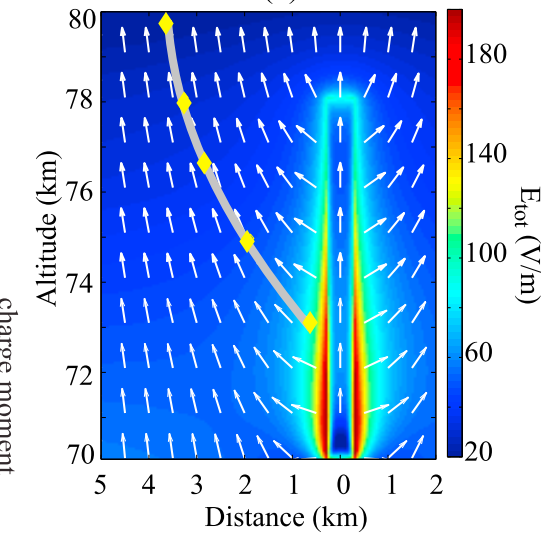

Figure 4. Estimated charge and electric fields for a sprite that occurred at 0343 UT on 13 August 2005. (a) Sprite images recorded at 7200 frames per second showing upward streamer development. The streamer tip locations are labeled with diamonds. (b) Measured azimuthal magnetic field. (c) Extracted lightning current moment and charge moment change waveforms. (d) Total electric fields in the vicinity of the bright core with $Q_{\text {core }}=-0.02 \mathrm{C}$. The color intensity represents the amplitude of the electric fields. The arrow lines represent the opposite direction of the total electric field (or motion direction of negative streamers). A streamline from the streamer initiation location is drawn as a reference.

[27] In our simulations, sprite core altitude extent is an important parameter determined from the images. Core radius is not essential and can be used to check the consistency of the inferred sprite core charge. Figure 6 shows the normalized electric fields in the radial direction (Figure 6a), vertical direction (Figure $6 \mathrm{~b}$ ) and the total electric fields (Figure 6c) produced by the lightning discharge and the sprite core with a total charge of $-0.02 \mathrm{C}$ over the entire $300 \mathrm{~m}$ radius. In Figure 6a, a normalized field of $E_{r} / E_{k}=0.9$ is observed at the surface throughout the entire altitude range of the sprite core. It should be noted that at a location directly above the lightning discharge, the horizontal field produced by the lightning is close to zero. Thus the horizontal electric fields are mainly produced by the charges in sprites. Figure $6 \mathrm{~b}$ shows the normalized fields in the vertical direction. A maximum normalized field about $1.2 E_{k}$ appears at the upper end of the cylinder. Luque and Ebert [2010] has pointed out that an ionization wave will be launched at the location where the field exceeds the breakdown field. However, this effect is not included in our results due to the electrostatic model applied. Figure $6 \mathrm{c}$ shows the total electric fields. The maximum total electric fields appear at the surface of the sprite core and slightly vary from 1 to $1.2 E_{k}$. This value is consistent with the total field at the surface of the glowing trail reported by Luque and Ebert [2010] and slightly greater than the spectroscopic measurements reported by
Morrill et al. [2002]. However, this is still reasonable since the spectroscopic measurement can be the field inside the streamer, where plenty of electrons exist. Both Liu [2010] and Luque and Ebert [2010] have reported that the total field inside is approximately $E_{k}$ along most of the glowing trail and quickly decay at the bottom part to the transition point. Interestingly, despite the fact that our simple electrostatic model does not include the ionization wave and uses an approximate charge distribution profile, a similar pattern has been observed in Figure 6c. The total field is more than $0.5 \mathrm{Ek}$ in the upper half of the bright core and quickly decreases at the bottom part. The consistency between our measurement-based results with independent results from numerical simulations validates the approach. The strong field close to $E_{k}$ at the outer surface also suggests that the persistent optical emission of the bright core is likely caused by the local electric fields instead of chemical processes. Although, in a streamer, the strong optical emissions do not necessarily appear at the high field regions, the electric field level does indicate the overall brightness of the streamer [Liu and Pasko, 2004; Liu et al., 2009a].

\subsubsection{Measurement Sensitivity}

[28] As mentioned earlier, we have chosen an altitudedependent charge distribution with the same scale height as air density. According to the simulation results reported by Luque and Ebert [2010], the charge amount along the alti- 
(a)

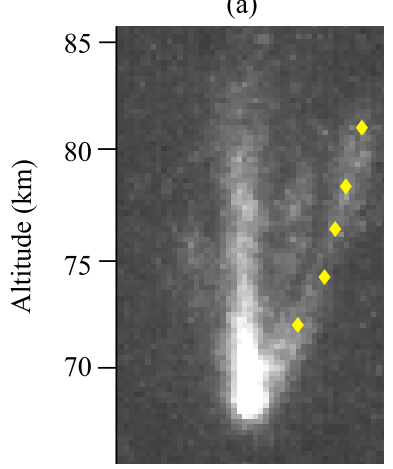

$\mathrm{Q}=-1.1 \times 10^{-2} \mathrm{C}$

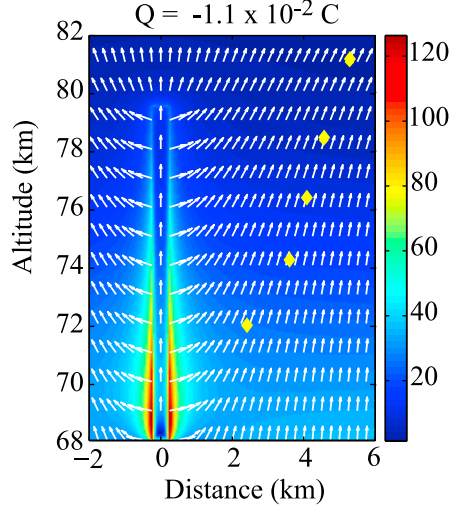

(b)

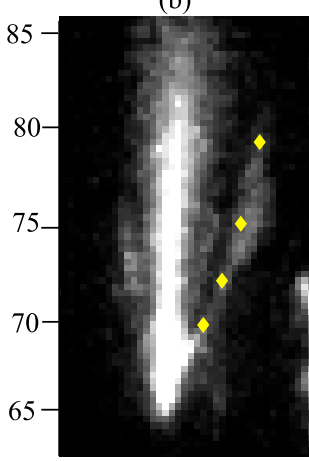

$\mathrm{Q}=-1.6 \times 10^{-2} \mathrm{C}$

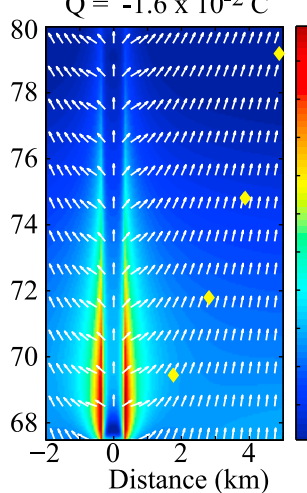

(c)
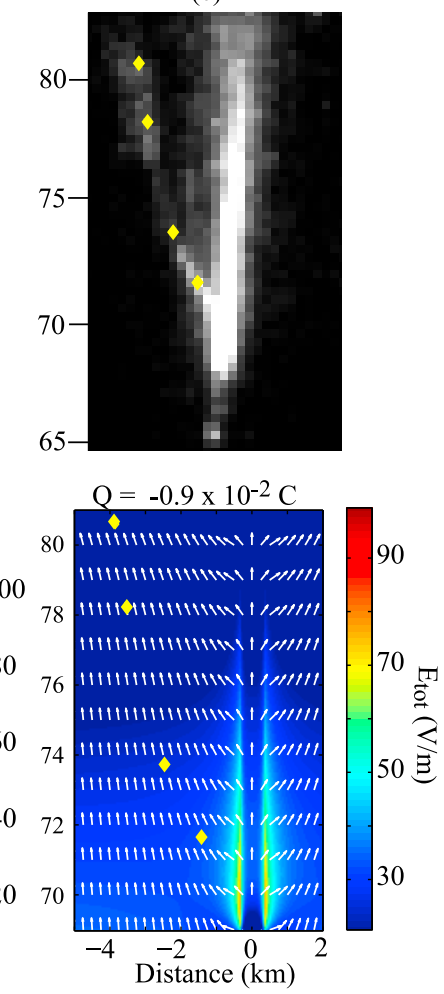

Figure 5. Streamer development history and simulated electric field for three dim sprites. (top) Streamer development history. (bottom) Amplitude and opposite direction of total electric fields.

tude is between uniform and an exponential decay with an inverse scale height of $0.25 \mathrm{~km}^{-1}$. Here we examine the uncertainty of the estimated charge amount caused by applying different vertical profiles. Figure 7 shows the amplitude and the direction of total electric fields for three vertical charge distributions and estimated amount of charge. In Figure $7 \mathrm{a}$, the amount of negative charge is $-0.018 \mathrm{C}$ and is uniformly distributed along the bright core. Figure $7 \mathrm{~b}$ shows the results of exponential charge distribution with an inverse scale height of $0.15 \mathrm{~km}^{-1}$ and a total amount charge of $-0.02 \mathrm{C}$. Figure $7 \mathrm{c}$ shows the results of exponential charge distribution with an inverse scale height of $0.25 \mathrm{~km}^{-1}$ and a total amount charge of $-0.03 \mathrm{C}$. For all the three simulation results, the total field direction agrees well with the upward streamer propagation direction. The three different charge amounts indicate that the inverse scale height of $0.15 \mathrm{~km}^{-1}$ could result in an under estimation by as much as $50 \%$. Thus, the amount of charge estimated with this distribution profile is a lower bound.

[29] For a fixed vertical distribution, we next examine the sensitivity of our measurement by analyzing how the total electric field direction is affected by the amount of charge in the bright core. Figure 8a again shows the development history of the upward streamer in Figure 4 but in a single image. Figures $8 \mathrm{~b}, 8 \mathrm{c}$, and $8 \mathrm{~d}$ show the total electric field caused by the same lightning discharge but three different amount of charge in the bright core region. Figure $8 \mathrm{~b}$ again shows the local electric field with the estimated $Q_{\text {core }}=$ $-0.02 \mathrm{C}$, in which the streamer propagation direction agree well with the opposite local electric field direction. In the results of Figures $8 \mathrm{c}$ and $8 \mathrm{~d}$, we varied $Q_{m c o r e}$ up and down by a factor of four to test the sensitivity between the field direction and the amount of charge in the core. It is clear that electric fields from neither of the two settings match the observed direction of streamer propagation. The field direction is too vertical for $Q=-0.005 \mathrm{C}$ and is too horizontal for $Q=-0.08 \mathrm{C}$. This indicates that the total electric field direction is sensitive to the total charge in the core, and our approach can be used to reliably infer the total sprite core charge by significantly less than a factor of four. However, several observational uncertainties can also influence
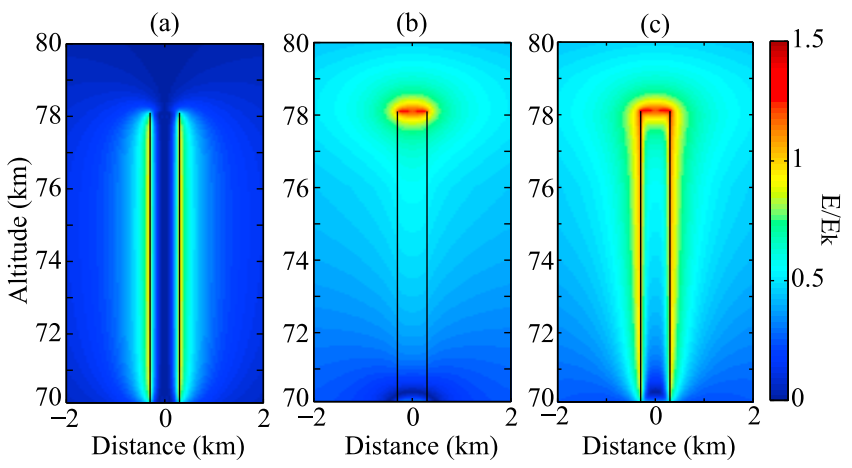

Figure 6. Normalized electric fields $\left(E / E_{k}\right)$ caused by lightning and sprite. (a) Electric fields in the horizontal direction. (b) Electric fields in the vertical direction. (c) Total electric fields. 
(a)

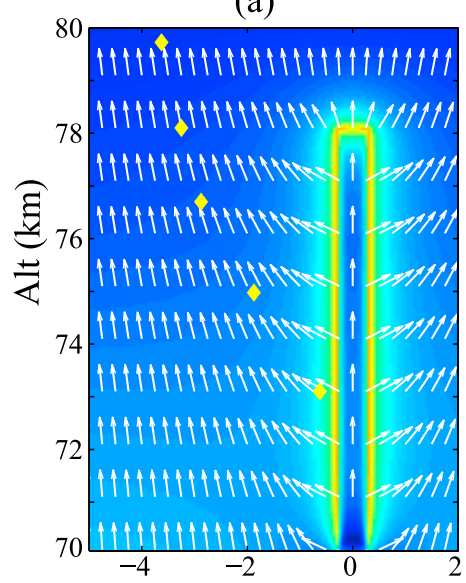

(b)

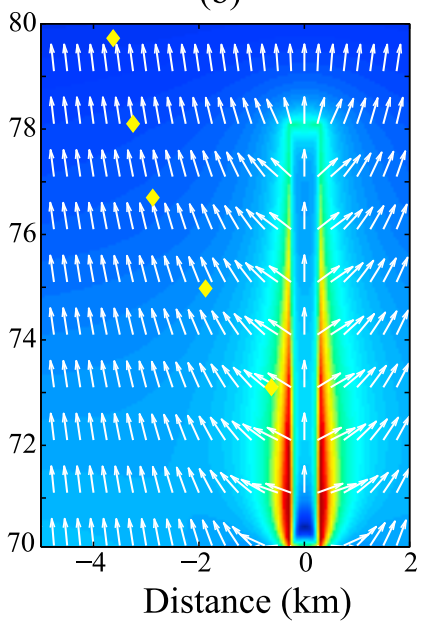

(c)

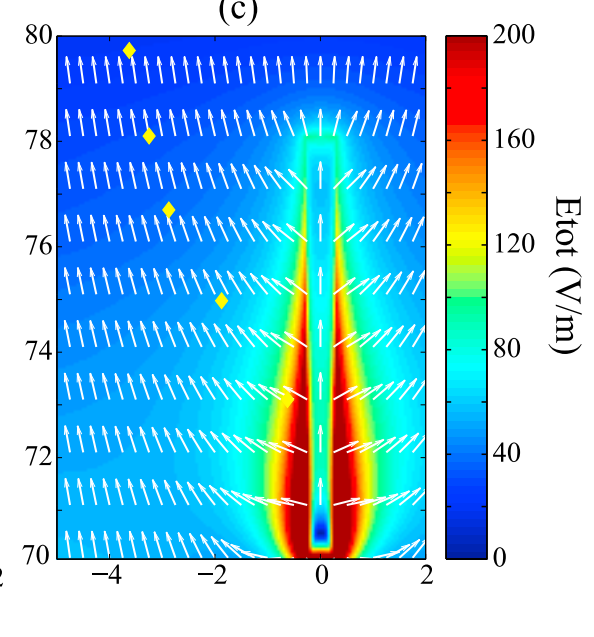

Figure 7. Total electric fields caused by the bright core with different vertical charge distributions. (a) Uniform charge distribution with $Q=-0.018$ C. (b) Exponential charge distribution (inverse scale height $=0.15 \mathrm{~km}^{-1}$ ) with $Q=-0.02 \mathrm{C}$. (c) Exponential charge distribution (inverse scale height $=$ $0.25 \mathrm{~km}^{-1}$ ) with $Q=-0.03 \mathrm{C}$.

the results. The first is the measured altitude of the sprite core. In our model, the entire altitude extent of the sprite core is measured from the high-speed images. On those images, the bottom boundary of the bright core is sharp and can be clearly determined. The very top region of the bright core is much dimmer and diffuse [Pasko and Stenbaek-Nielsen, 2002], which is not included in the charge estimation. Thus, in Figures 2 and 4, the $8 \mathrm{~km}$ height is the lower bound for the charge region. Although the altitude extent of the sprite core is used in the calculation, the resulting total sprite core charge does not depend sensitively on it. In our model, the simulation result is not very sensitive to the maximum altitude of the bright core. This is because most of the charge is in the bottom part of the cylinder due to the exponential charge distribution profile applied. For example, in Figure 4, if we increase the height of the cylinder by $50 \%$ (to $12 \mathrm{~km}$ ) but keep the bottom and the total charge amount the same, the horizontal fields at the streamer locations vary about $10 \%-30 \%$. Thus, excluding the dimmer and diffuse top region does not significantly change the estimated amount of charge. When estimating the sprite core altitude, we assumed that sprites occurred directly above their parent lightning discharge. The unknown offset between them (perhaps a few tens of $\mathrm{km}$ [Wescott et al., 2001]) contributes an overall altitude uncertainty of approximately $\pm 3 \mathrm{~km}$. This uncertainty is constant in the entire altitude extent of the bright core. By shifting the entire core up or down by $3 \mathrm{~km}$, our results show that the estimated amount of charge can vary up to $50 \%$.

[30] The second is the viewing angle. Due to the fact the propagation direction of upward streamers on the highspeed images is actually the 2-D projection of a 3-D sprite, the horizontal component of upward streamer propagation can be greater than appearing on the images, which implies a higher field in horizontal direction and more charge in the upper bright core. These limitations can only be removed by triangulation of sprite locations. It should be noted that the above estimated charge amount in the bright core is at the stage where upward streamer become fully developed. This is close to the stage of sprite maximum brightness. The charge motion after the analyzing time is not included. Also during the propagation of upward streamers, we have assumed that the charge distribution in the core is static and does not change. In reality, downward streamers may still develop and introduce moving charges in the bright core and upward streamers. This may introduce an error in the estimated amount of charge. However, due to the fact that the size and shape of the bright core do not vary appreciably, the (a)

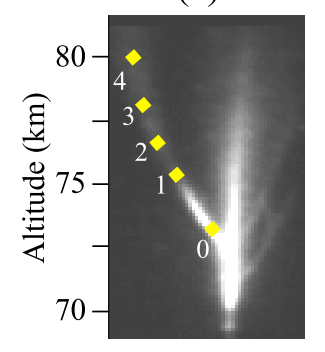

(c)

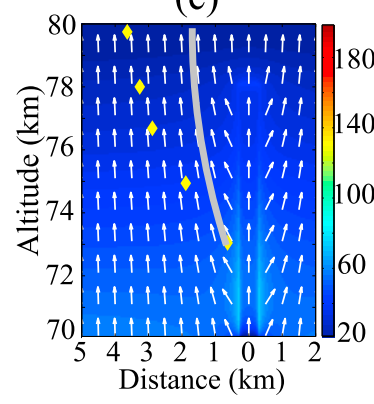

(b)

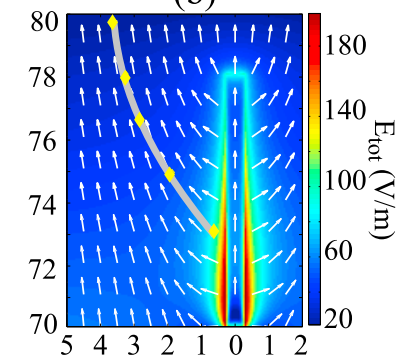

(d)

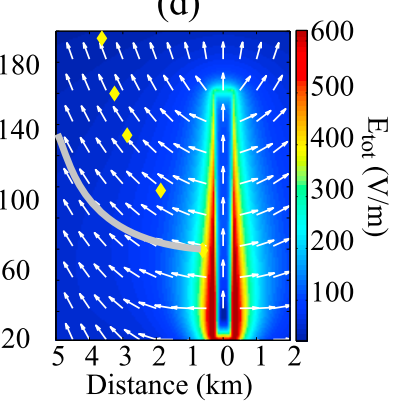

Figure 8. (a) Development history of the upward streamer in a single image. (b-d) The amplitudes and opposite directions of total electric fields with $Q_{\text {core }}=-0.02,-0.005$, and $-0.08 \mathrm{C}$, respectively. The streamer tip locations are represented by diamonds. A streamline from the streamer initiation location is drawn as a reference in each plot. 


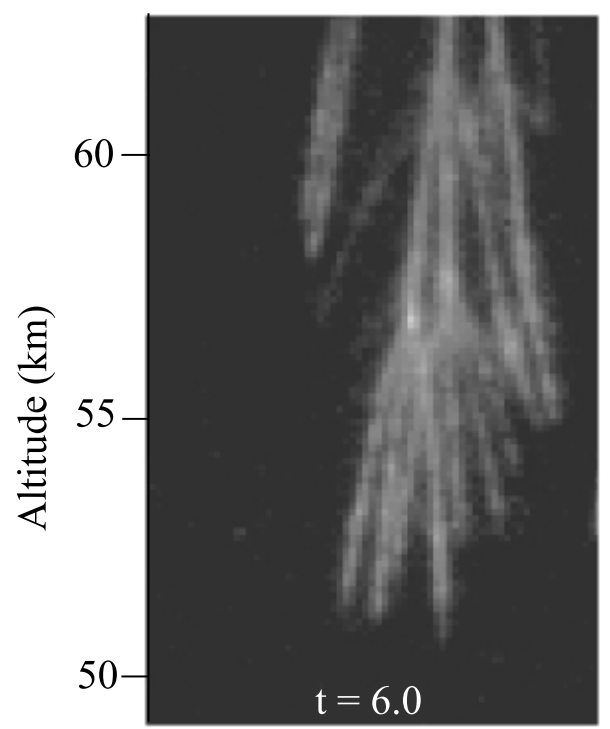

Figure 9. Image of downward streamers for sprite detected at 0343 UT on 13 August 2005. There are a total of 11 downward streamers terminating between 50 and $60 \mathrm{~km}$ altitudes.

amount of these moving charges should be much smaller comparing to the amount of existing charge in the bright core.

\subsubsection{Estimated Downward Streamer Charge}

[31] Because the sprite cores develop as the top part of the downward moving positive streamers in sprites [Stanley et al., 1999; Cummer et al., 2006; Stenbaek-Nielsen and McHarg, 2008], it is physically plausible to interpret the negative sprite core charge as the source of at least some of the positive charge in the downward streamers. Section 3.4 above discussed the implications of the degree of electrical connection of the sprite core to the ionosphere and showed that this connection cannot be too strong, otherwise negative space charge could not persist. Moreover, the subsequent upward streamers in sprites generally form in bright, fast moving sprites [Cummer et al., 2006], which suggests the possibility that these upward streamers form because the downward positive streamers require more charge than the sprite core can provide, and the subsequent negative streamers are how the system can provide more positive charge to the lower-altitude sprite features. These streamers are further evidence that the electrical connection between the sprite core and the ionosphere is not strong. If it were, the ionosphere could provide any needed charge and the negative streamers would not be needed.

[32] We acknowledge that some positive charge to the downward streamers may be supplied by the ionosphere. However, if we assume that sprites are not connected to the conducting ionosphere, we can obtain a lower bound on the average charge in each downward streamer simply through counting the downward streamer tips and combining the result with the inferred sprite core charge. Figure 9 shows the bottom part of the first sprite analyzed. There are a total of 11 discernable downward streamers terminating in the $50-60 \mathrm{~km}$ altitude range. Thus the average charge in each downward streamer tip is about $0.02 / 11=1.8 \times 10^{-3} \mathrm{C}$. It should be emphasized again that if the sprite is partially connected to the ionosphere (as shown in Figure 3d), this estimated amount of charge in the bright core and downward streamers is the lower bound due to the positive charges provided by the ionosphere. Based on the number of observed downward streamers at the time of the upward streamer in all four events analyze here, the estimated lower bound on the charge in each downward streamer at full development varies over the relatively small range of 1.8 $5.5 \times 10^{-3} \mathrm{C}$. These calculations for each observed sprite are summarized below in Table 2 .

\subsection{Bright Sprites}

[33] Applying the above approach to big and bright sprites is more challenging because individual upward streamers are not always easy to identify, and downward streamers can be too numerous to easily count. However, sprites with strong optical emissions are sometimes accompanied with detectable radio emissions, which allows us to estimate the total charge transfer in sprites by extracting the sprite current and charge moment waveforms [Cummer et al., 1998]. In this section we apply both of these approaches to estimate the charge in two bright sprites.

[34] Figure 10 shows a close-up of a bright, multielement sprite recorded on 13 August 2005 at 0414 UT. There are approximately 10 individual sprite elements at the time of maximum brightness, but only one of them produced any subsequent upward streamers at the time of maximum brightness. The total lightning charge moment change at the time of peak brightness was $400 \mathrm{C} \mathrm{km}$. In this event, the height of the bright core is about $8 \mathrm{~km}$ with the bottom at $70 \mathrm{~km}$ altitude. The measured core radius is about $440 \mathrm{~m}$. Figure $10 \mathrm{~b}$ shows the total electric field in the vicinity of the

Table 2. Summary of the Six Events Analyzed ${ }^{\mathrm{a}}$

\begin{tabular}{|c|c|c|c|c|c|c|c|c|c|}
\hline $\begin{array}{l}\text { Event } \\
\text { Number }\end{array}$ & $\begin{array}{c}\text { CMC } \\
(\mathrm{C} \mathrm{km})\end{array}$ & $\begin{array}{c}\text { Upward Streamer } \\
\text { Angle (deg) }\end{array}$ & $h_{\text {core }}(\mathrm{km})$ & $r_{\text {core }}(\mathrm{m})$ & $Q_{\text {core }}(\mathrm{C})$ & $\begin{array}{c}\text { Downward Streamer } \\
\text { Number }\end{array}$ & Average $Q_{\text {down }}(\mathrm{C})$ & $\begin{array}{l}\text { Sprite } \\
\text { Current }\end{array}$ & $\begin{array}{c}Q(\mathrm{C}) \\
\text { (Sprite Current) }\end{array}$ \\
\hline 1 & 300 & 30 & 12 & $<350$ & -0.009 & 3 & $3.0 \times 10^{-3}$ & no & N/A \\
\hline 2 & 330 & 31 & 11 & 240 & -0.011 & 2 & $5.5 \times 10^{-3}$ & no & $\mathrm{N} / \mathrm{A}$ \\
\hline 3 & 330 & 28 & 13 & $<350$ & -0.016 & 4 & $4.0 \times 10^{-3}$ & no & N/A \\
\hline 4 & 410 & 37 & 8 & 300 & -0.020 & 11 & $1.8 \times 10^{-3}$ & no & N/A \\
\hline 5 & 440 & 36 & 8 & 400 & -0.028 & 7 & $4.0 \times 10^{-3}$ & no & N/A \\
\hline 6 & 540 & 22 & $\sim 10$ & 420 & -0.034 & $>10$ & $3.4 \times 10^{-3}$ & yes & -0.08 \\
\hline
\end{tabular}

${ }^{a}$ Includes lightning charge moment change, upward streamer departure angle, bright core height and radius, total charge in the bright core estimated from upward streamers, number of downward streamers, average charge in each downward streamer, detectable radio emissions from the sprite (sprite current), and total charge estimated from the sprite current. The lightning charge moment change (CMC) is computed up to the time of the upward streamer development. 

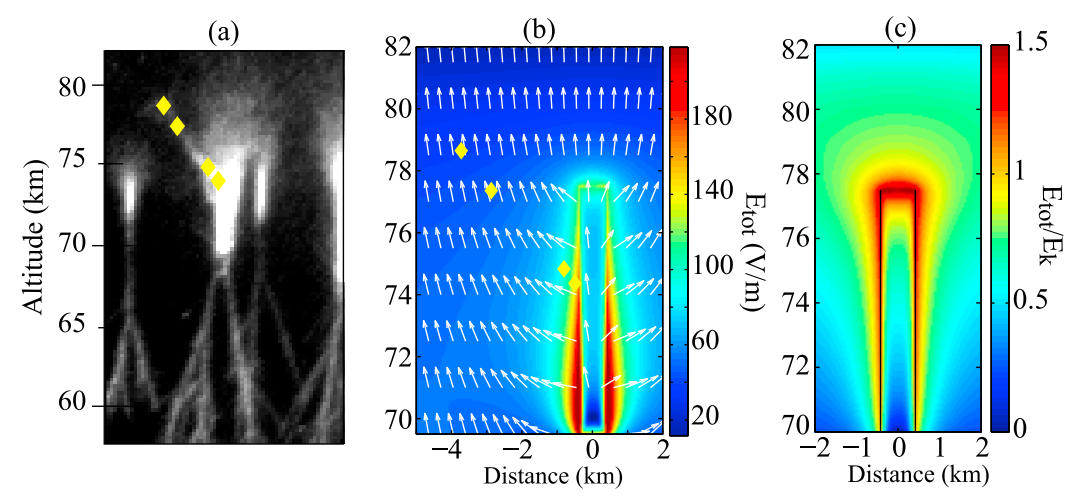

Figure 10. (a) Upward streamer development history for one of the sprite elements detected at 0414 UT on 13 August 2005. (b) Total electric field in the vicinity of the bright core with $Q=-0.028$ C. (c) Normalized total electric fields in the vicinity of the bright core.

bright core, and we infer a total sprite core charge of $-0.028 \mathrm{C}$. Figure 10c shows the normalized total electric field as a consistency check. At the surface of the cylinder, the maximum normalized electric field is about $1.1-1.3 E_{k}$, which is similar to the event shown in Figure 6. In this sprite element, there are seven distinct downward streamers observed. Thus the average charge in each downward streamer is $\sim 4 \times 10^{-3} \mathrm{C}$, still in excellent agreement with the simpler sprites analyzed in section 4.1. Lastly, we analyze in detail a sprite for which we can clearly detect sprite currents but still identify individual sprite elements from the images. Figure 11 shows a bright sprite detected on 13 August 2005 at 0325 UT. The high-speed image (Figure 11a) shows that there are at least 17 identifiable sprite elements, each with a single sprite core, in this bright sprite. Accounting for some elements that are probably obscured because they are in front of or behind the bright elements in the middle, we estimate that there are about 30 distinct cores in this sprite. Figure $11 \mathrm{~b}$ shows the remotely measured magnetic field in the azimuthal direction. This magnetic field includes the radiation from both lightning discharge and current flows inside the sprite body. The two black lines represent the time of sprite initiation and maximum brightness. The second peak in the measured magnetic field is the radiation from the sprite. After applying the deconvolution technique, we separate the current moment and charge moment history from the two different sources. Figure $11 \mathrm{c}$ shows the lightning current moment and charge moment change. The impulse charge moment change of the lightning discharge is close to $500 \mathrm{C} \mathrm{km}$, which is consistent with the observed bright and short delayed sprite [Hu et al., 2007]. Figure 11d shows the current moment waveform of current flows in the sprite. At the time of maximum sprite brightness, the total sprite charge moment change is about $75 \mathrm{C} \mathrm{km}$. Since the downward streamers on average travel from $\sim 75 \mathrm{~km}$ to $\sim 45 \mathrm{~km}$ altitude, and spreading this charge equally over 30 cores, the average charge transfer in each sprite element over this distance is about $-0.08 \mathrm{C}$. If no charge is supplied by the ionosphere, this is also the average negative charge present in a single sprite core.

[35] We now estimate the same value from the upward streamer directions. Figure 12a shows the upward streamer development history for a sprite element. From the highspeed images, the upward streamer travels from 69 to $73 \mathrm{~km}$ altitude. The bottom of the bright core is at $67 \mathrm{~km}$ altitude. The top of the bright core cannot be clearly determined due to the fact that it merged into optical emissions from other sprite elements, but lies between 74 and $78 \mathrm{~km}$. However, as mentioned earlier, the total amount charge in the bright core is not very sensitive to the height of the bright core. Thus we choose a length of $10 \mathrm{~km}$, which falls in the above range and is a typical value for the length of the bright core.

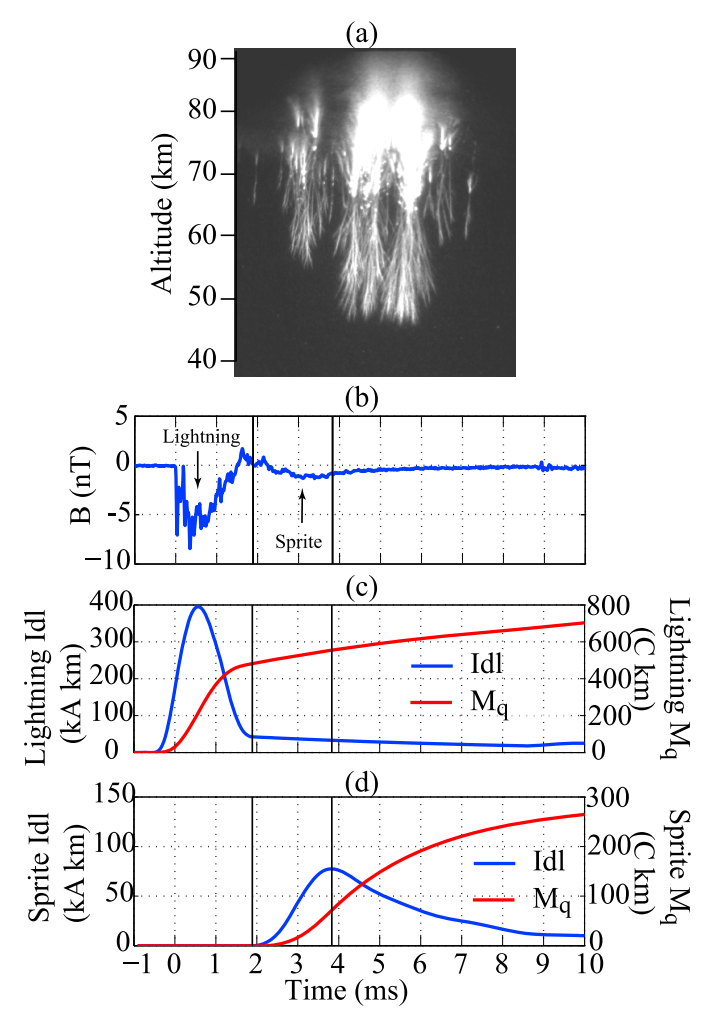

Figure 11. (a) High-speed video for a bright sprite detected at 0325 UT on 13 August 2005. (b) Measured azimuthal magnetic field. (c) Current moment and charge moment change history of the sprite-producing lightning. (d) Current moment and charge moment change history of the sprite. The black lines represent the time of sprite initiation and maximum brightness. 


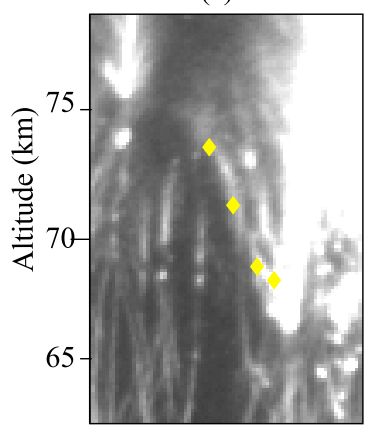

(b)

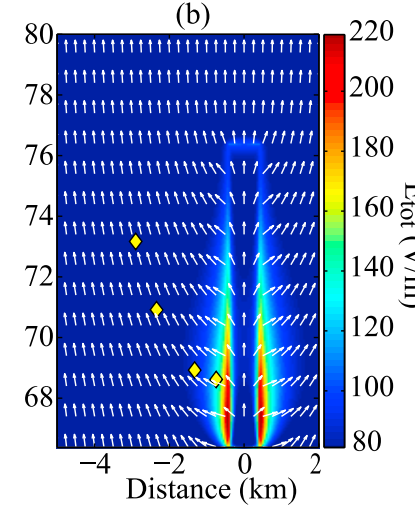

(c)

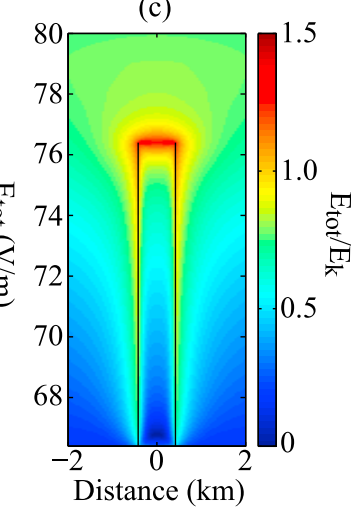

Figure 12. (a) Upward streamer development history for one sprite element. (b) Total electric field in the adjacent region of the bright core with $Q=-0.034$ C. (c) Normalized total electric field.

The radius of the bright core is $420 \mathrm{~m}$ measured from highspeed images. A best match between the streamer propagation direction and the total electric field is achieved when the total sprite core charge is $-0.034 \mathrm{C}$. This is within approximately a factor of 2.5 of the completely independent estimate obtained from the sprite current measurements. Figure $12 \mathrm{~b}$ shows the amplitude and direction of the total electric field. A consistency check is also performed by looking at the normalized electric field at the boundary of the $3-\mathrm{D}$ cylinder. In this event, the maximum normalized electric field $\left(E_{t o t} / E_{k}\right)$ is $0.75-1.1 E_{k}$ at $\mathrm{r}=420 \mathrm{~m}$, perfectly in line with expectations from the events shown in Figures 6 and 10. Again, the high field level at the surface of the bright core indicates that the persistent optical emission is likely produced by instantaneous electric fields instead of longduration chemical processes. Due to the complexity of the sprite structure, the number of downward streamers in this sprite element cannot be accurately determined when merging with streamers from other sprite elements. However, at least 10 downward streamers can be identified in the images. Thus the average charge amount in each downward streamer is about $3.4 \times 10^{-3} \mathrm{C}$, still completely in line with the estimated charge per positive streamer in the other events.

\subsection{Measurement Summary}

[36] Here we summarize our results for all the six events analyzed in Table 2. These events are listed in the order of increasing lightning charge moment change at the time of the upward streamer development, which is a good indicator of the background lightning-driven electric field. These charge moment changes are consistent with results reported by $\mathrm{Hu}$ et al. [2007] for short delayed [Li et al., 2008] dim and bright sprites. Table 2 immediately reveals some connections between different features. Except for the last event, the upward streamer departure angles are close to 30 35 degrees from vertical. This indicates at the location of streamer tips, the horizontal electric field produced by the bright core is about 60 .

[37] The radius of the bright core also appears to be related to the amount of charge inside, with a larger radius implying more total charge. It should be emphasized that the radius in events 1 and 3 are probably overestimated due to the spatial resolution of the image. More downward streamers from a single sprite core are also observed in those events with more charge in the core. This indicates that the amount of charge in the bright core is also connected to the branching of downward streamers. This in turn is consistent with the idea that the downward streamers dictate how much charge is pulled from the sprite core and whether or not subsequent upward negative streamers are required to supply this charge.

\section{Conclusions}

[38] In this work, we have estimated the total electric charge in sprites using a measurement-based approach that builds on model and measurement based estimates of the lightning-driven background electric fields in the mesosphere and observations from high-speed video of the propagation direction of upward streamers that initiate from the bright core region of sprites. Assuming the streamers propagate along the direction of local electric fields, and that the local electric fields are modified by space charge present in the sprite core, we have inferred the total electric charge in the observed sprite cores that is consistent with the observed streamer propagation directions.

[39] In six separate sprites we find that individual bright sprite cores contain significant negative space charge that ranges between $-0.009 \mathrm{C}$ to $-0.034 \mathrm{C}$. Accounting for the uncertainties caused by using different vertical charge distributions, these values could be as much as $50 \%$ higher. Detailed electrostatic simulations show that this amount of charge, when spread over the observed core sizes of several hundred meters in diameter and approximately $10 \mathrm{~km}$ in length, results in a local electric field at the outer edges of the sprite core close to the breakdown field. This is consistent with the recent simulation results reported by Luque and Ebert [2010] and provides an independent validation of our inferred charges. Moreover, this indicates that the relatively long duration (several milliseconds) optical emissions typically observed from sprite cores [Cummer et al., 2006] are driven predominantly by instantaneous local electric fields and not by long-duration chemistry initiated by previous energy input.

[40] Given this significant negative charge, we interpret the sprite core region as the partial and perhaps dominant sink of the negative charge created by the downward positive polarity streamers. This notion is supported by numerical 
simulations that show that only a weak or nonexistent electrical connection between the ionosphere and the downward streamer results in significant negative charge at the top of the streamer channel. This further suggests that subsequent negative polarity upward streamers that appear in some sprites a few milliseconds after initiation are created when the downward positive sprite streamers require more charge than can be supplied by this sprite core. This is consistent with observations that show the subsequent upward streamers are not always present, especially in smaller sprites.

[41] Assuming that the sprite core is the source of all of the positive charge in the downward streamers, we find that the average downward streamer contains a charge of at least $2-4 \times 10^{-3} \mathrm{C}$. Observed sprite current in one bright sprite suggests a positive charge in each distinct downward streamer that is only 2.5 times larger than this lower bound. This indicates that our estimate of the positive charge in downward sprite streamers and the negative charge in the sprite core is physically reasonable, and also that the charge motion from the sprite core to the downward streamers is a likely source of sprite current. Although our detailed results are partially influenced by the assumptions in the electrostatic model and the uncertainties in the measurements, the primary conclusions regarding the charge magnitude and the origin of the sprite core optical emission are not significantly affected. This work can be a step to further constrain the detailed physical and chemical models that can predict the impact of sprites on the mesosphere.

[42] Acknowledgments. Robert Lysak thanks the reviewers for their assistance in evaluating this paper.

\section{References}

Briels, T. M. P., J. Kos, G. J. J. Winands, E. M. van Veldhuizen, and U. Ebert (2008), Positive and negative streamers in ambient air: Measuring diameter, velocity and dissipated energy, J. Phys. D Appl. Phys., 41, 234004, doi:10.1088/0022-3727/41/23/234004.

Cummer, S. A. (2003), Current moment in sprite-producing lightning, J. Atmos. Sol. Terr. Phys., 65, 499-508.

Cummer, S. A., and U. S. Inan (1997), Measurement of charge transfer in sprite-producing lightning using ELF radio atmospherics, Geophys. Res. Lett., 24(14), 1731-1734, doi:10.1029/97GL51791.

Cummer, S. A., and W. A. Lyons (2005), Implications of lightning charge moment changes for sprite initiation, J. Geophys. Res., 110, A04304, doi:10.1029/2004JA010812.

Cummer, S. A., U. S. Inan, T. F. Bell, and C. P. Barrington-Leigh (1998), ELF radiation produced by electrical currents in sprites, Geophys. Res Lett., 25(8), 1281-1284, doi:10.1029/98GL50937.

Cummer, S. A., N. Jaugey, J. Li, W. A. Lyons, T. E. Nelson, and E. A. Gerken (2006), Submillisecond imaging of sprite development and structure, Geophys. Res. Lett., 33, L04104, doi:10.1029/2005GL024969.

Ebert, U., C. Montijn, T. M. P. Briels, W. Hundsdorfer, B. Meulenbroek, A. Rocco, and E. M. van Veldhuizen (2006), The multiscale nature of streamers, Plasma Sources Sci. Technol., 15, s118-s129.

Gerken, E. A., U. S. Inan, and C. P. Barrington-Leigh (2000), Telescopic imaging of sprites, Geophys. Res. Lett., 27(17), 2637-2640, doi:10.1029/ 2000 GL000035.

Hu, W., S. A. Cummer, W. A. Lyons, and T. E. Nelson (2006), An FDTD model for low and high altitude lightning-generated EM fields, IEEE Trans. Antennas Propag., 54(5), 1513-1522.

$\mathrm{Hu}$, W., S. A. Cummer, and W. A. Lyons (2007), Testing sprite initiation theory using lightning measurements and modeled electromagnetic fields, J. Geophys. Res., 112, D13115, doi:10.1029/2006JD007939.

Li, J., and S. A. Cummer (2009), Measurement of sprite streamer acceleration and deceleration, Geophys. Res. Lett., 36, L10812, doi:10.1029/ 2009GL037581.
Li, J., S. A. Cummer, W. A. Lyons, and T. E. Nelson (2008), Coordinated analysis of delayed sprites with high-speed images and remote electromagnetic fields, J. Geophys. Res., 113, D20206, doi:10.1029/2008JD010008.

Liu, N. (2010), Model of sprite luminous trail caused by increasing streamer current, Geophys. Res. Lett., 37, L04102, doi:10.1029/ 2009GL042214

Liu, N., and V. P. Pasko (2004), Effects of photoionization on propagation and branching of positive and negative streamers in sprites, J. Geophys. Res., 109, A04301, doi:10.1029/2003JA010064.

Liu, N., and V. P. Pasko (2005), Molecular nitrogen LBH band system far-UV emissions of sprite streamers, Geophys. Res. Lett., 32, L05104, doi:10.1029/2004GL022001.

Liu, N., V. P. Pasko, H. U. Frey, S. B. Mende, H.-T. Su, A. B. Chen, R.-R. Hsu, and L.-C. Lee (2009a), Assessment of sprite initiating electric fields and quenching altitude of a ${ }^{1} \Pi_{g}$ state of $\mathrm{N} 2$ using sprite streamer modeling and ISUAL spectrophotometric measurements, J. Geophys. Res., 114, A00E02, doi:10.1029/2008JA013735.

Liu, N. Y., V. P. Pasko, K. Adams, H. C. Stenbaek-Nielsen, and M. G. McHarg (2009b), Comparison of acceleration, expansion, and brightness of sprite streamers obtained from modeling and high-speed video observations, J. Geophys. Res., 114, A00E03, doi:10.1029/2008JA013720.

Luque, A., and U. Ebert (2009), Emergence of sprite streamers from screening-ionization waves in the lower ionosphere, Nat. Geosci., 2, 757-760, doi:10.1038/NGE0662.

Luque, A., and U. Ebert (2010), Sprites in varying air density: Charge conservation, glowing negative trails and changing velocity, Geophys. Res. Lett., 37, L06806, doi:10.1029/2009GL041982.

Luque, A., V. Ratushnaya, and U. Ebert (2008), Positive and negative streamers in ambient air: Modeling evolution and velocities, J. Phys. D Appl. Phys., 41, 234005, doi:10.1088/0022-3727/41/23/234005.

McHarg, M. G., H. C. Stenbaek-Nielsen, and T. Kammae (2007), Observations of streamer formation in sprites, Geophys. Res. Lett., 34, L06804, doi:10.1029/2006GL027854.

Morrill, J. S., E. J. Bucsela, V. P. Pasko, S. L. Berg, M. J. Heavner, D. R. Moudry, W. M. Benesch, E. M. Wescott, and D. D. Sentman (1998), Time resolved N2 triplet state vibrational populations and emissions associated with red sprites, J. Atmos. Sol. Terr. Phys., 60, 811-829.

Morrill, J. S., et al. (2002), Electron energy and electric field estimates in sprites derived from ionized and neutral N2 emissions, Geophys. Res. Lett., 29(10), 1462, doi:10.1029/2001GL014018.

Pasko, V. P. (2007), Red sprite discharges in the atmosphere at high altitude: The molecular physics and the similarity with laboratory discharges, Plasma Sources Sci. Technol., 16, s13-s29, doi:10.1088/0963-0252/16/1/S02.

Pasko, V. P., and H. C. Stenbaek-Nielsen (2002), Diffuse and streamer regions of sprites, Geophys. Res. Lett., 29(10), 1440, doi:10.1029/ $2001 \mathrm{GL} 014241$

Pasko, V. P., U. S. Inan, and T. F. Bell (1997), Sprite produced by quasielectrostatic heating and ionization in the lower ionosphere, J. Geophys. Res., 102(A3), 4529-4561, doi:10.1029/96JA03528.

Raizer, Y., G. Milikh, M. Shneider, and S. Novakovski (1998), Long streamer in the upper atmosphere above thundercloud, J. Phys. D Appl. Phys., 31, 3255-3264.

Sentman, D. D. (1995), Preliminary results from the Sprites94 aircraft campaign: 1. Red sprite, Geophys. Res. Lett., 22(10), 1205-1208, doi:10.1029/95GL00583.

Sentman, D. D., H. C. Stenbaek-Nielsen, M. G. McHarg, and J. S. Morrill (2008), Plasma chemistry of sprite streamers, J. Geophys. Res., 113, D11112, doi:10.1029/2007JD008941.

Stanley, M. S., P. Krehbiel, M. Brook, C. Moore, W. Rison, and B. Abrahams (1999), High-speed video of initial sprite development, Geophys. Res. Lett., 26(20), 3201-3204, doi:10.1029/1999GL010673.

Stenbaek-Nielsen, H., and M. G. McHarg (2008), High time-resolution sprite imaging: Observations and implications, J. Phys. D Appl. Phys., 41, 234009.

Stenbaek-Nielsen, H., D. R. Moudry, E. M. Wescott, D. D. Sentman, and F. T. S. Sabbas (2000), Sprites and possible mesospheric effect, Geophys. Res. Lett., 27(23), 3829-3832, doi:10.1029/2000GL003827.

Stenbaek-Nielsen, H., M. G. McHarg, T. Kanmae, and D. D. Sentman (2007), Observed emission rates in sprite streamer heads, Geophys. Res. Lett., 34, L11105, doi:10.1029/2007GL029881.

Wescott, E. M., H. C. Stenbaek-Nielsen, D. D. Sentman, M. J. Heavner, D. R. Moudry, and F. T. S. Sabbas (2001), Triangulation of sprites, associated halos and their possible relation to causative lightning and micrometeors, J. Geophys. Res., 106(A6), 10,467-10,477, doi:10.1029/2000JA000182.

S. Cummer and J. Li, Department of Electrical and Computer Engineering, Duke University, Durham, NC 27708, USA. (j1108@duke.edu) 